\title{
Prophylactic effects of Lonicera japonica extract on dextran sulphate sodium-induced colitis in a mouse model by the inhibition of the Th1/Th17 response
}

\author{
Jae-Woo Park ${ }^{1}$, Hyunsu Bae ${ }^{1}$, Gihyun Lee ${ }^{1}$, Beom-Gi Hong ${ }^{1}$, Hye Hyun Yoo ${ }^{2}$, Sung-Jig Lim³ ${ }^{3}$ \\ Kyungjin Lee ${ }^{1}$, Jinsung Kim ${ }^{1}$, Bongha Ryu ${ }^{1}$, Beom-Joon Lee ${ }^{4}$, Jinhyun Bae ${ }^{1}$, Hyejung Lee ${ }^{5}$ and \\ Youngmin $\mathrm{Bu}^{1 *}$ \\ ${ }^{1}$ College of Oriental Medicine, Kyung Hee University, Seoul 130-701, Republic of Korea \\ ${ }^{2}$ College of Pharmacy, Hanyang University, Ansan 426-791, Republic of Korea \\ ${ }^{3}$ Graduate School of Medicine, Kyung Hee University, Seoul 130-701, Republic of Korea \\ ${ }^{4}$ Kangnam Korean Hospital, Kyung Hee University, Seoul 135-501, Republic of Korea \\ ${ }^{5}$ Acupuncture and Meridian Science Research Center, College of Korean Medicine, Kyung Hee University, Seoul 130-701, \\ Republic of Korea
}

(Submitted 14 November 2011 - Final revision received 15 February 2012 - Accepted 27 February 2012 - First published online 9 May 2012)

\section{Abstract}

Inflammatory bowel diseases (IBD) are chronically relapsing inflammatory disorders of the intestine. Although some therapeutic agents, including steroids, are available for the treatment of IBD, these agents have limited use. Therefore, dietary supplements have emerged as possible interventions for IBD. Japanese honeysuckle flower, the flower of Lonicera japonica, is a well-known dietary supplement and has been used to prevent or treat various inflammatory diseases. In the present study, we investigated the effects of $L$. japonica on experimental murine colitis. Colitis was induced by $5 \%$ dextran sulphate sodium (DSS) in Balb/c mice. The water extract of $L$. japonica (LJE) at doses of 20,100 or $500 \mathrm{mg} / \mathrm{kg}$ was orally administered to mice twice per day for $7 \mathrm{~d}$. Body weight, colon length and a histological damage score were assessed to determine the effects on colitis. Cytokine profiles were assessed to examine the effects on helper $\mathrm{T}$ (Th) cell-related immunological responses. In addition, $\mathrm{CD} 4^{+} \mathrm{CD} 25^{+} \mathrm{Foxp} 3^{+} \mathrm{T}$ cells were analysed in vivo and in vitro for investigating the effects on regulatory $\mathrm{T}\left(\mathrm{T}_{\mathrm{reg}}\right)$ cells. LJE showed dose-dependent inhibitory effects against colon shortening, weight loss and histological damage. LJE down-regulated IL-1 $\beta$, TNF- $\alpha$, interferon- $\gamma$, IL-6, IL-12 and IL-17. However, LJE did not show any significant effects on IL-10, IL-23, transforming growth factor- $\beta 1$ and $\mathrm{T}_{\text {reg }}$ cell populations. In conclusion, LJE showed protective effects against DSS-induced colitis via the Th1/Th17 pathway and not via $\mathrm{T}_{\text {reg }}$ cell-related mechanisms.

Key words: Inflammatory bowel disease: Lonicera japonica: Dextran sulphate sodium: Regulatory T cells: Th1/Th17 pathway

Inflammatory bowel diseases (IBD) are defined as chronic inflammatory conditions of the intestine and are generally divided into Crohn's disease and ulcerative colitis ${ }^{(1)}$. The prevalence of IBD is increasing worldwide, with recent annual increases of about 42.1 and 439 patients per $10^{5}$ people in South Korea and North America, respectively ${ }^{(1)}$. Although current treatments, including non-steroidal anti-inflammatory drugs and corticosteroids, have been administered to reduce symptoms, they have potential side effects including hypersensitivity, adrenal suppression and lymphoma ${ }^{(2)}$.

Various diets or dietary ingredients such as fruits, vegetables and fibre are considered safe and are used to prevent the initiation or aggravation of gut inflammation through immune interactions and the alteration of the gut microbiota ${ }^{(3)}$. Therefore, many studies have been performed on the use of dietary supplements and natural products as alternative therapies for $\operatorname{IBD}^{(4,5)}$.

Japanese honeysuckle flower (Lonicera japonica Thunb.; LJ) is a well-known dietary supplement that has long been used in $\mathrm{Asia}^{(6)}$. LJ is the main ingredient of the herbal formula 'Bojanggunbi-tang', which has widely been used to treat IBD in Korea and has demonstrated protective effects against dextran sulphate sodium (DSS)- and trinitrobenzene sulfonic acid-induced colitis in mice ${ }^{(7)}$. LJ has been reported to

Abbreviations: CGA, chlorogenic acid; DSS, dextran sulphate sodium; IBD, inflammatory bowel disease; IFN- $\boldsymbol{\gamma}$, interferon- $\gamma$; LJ, Lonicera japonica; LJE, water extract of Lonicera japonica; TGF- $\beta 1$, transforming growth factor- $\beta 1$; $\mathrm{T}_{\text {reg, }}$, regulatory $\mathrm{T}$ cell

*Corresponding author: Y. Bu, fax +82 2964 0325, email ymbu@khu.ac.kr 
have various bioactivities including anti-inflammatory ${ }^{(8)}$, anti-atherogenic ${ }^{(9)}$, anti-carcinogenic ${ }^{(10)}$, analgesic ${ }^{(11)}$ and neuroprotective $^{(12)}$ effects. It contains various compounds including chlorogenic acid (CGA), luteolin, ferulic acid, quercetin and caffeic acid that are responsible for these effects $^{(8-12)}$. To our knowledge, the effect of LJ on IBD has not yet been reported.

Of the many existing animal models of IBD, chemically induced models including DSS- and trinitrobenzene sulfonic acid-induced colitis are among the most common ${ }^{(13)}$. DSSinduced murine colitis is well characterised by clinical symptoms (diarrhoea, bloody stools, weight loss and colon shortening), histological changes (cryptal damages, erosion, ulceration and infiltration of the inflammatory cells) and changes in inflammatory mediators including chemokines and cytokines ${ }^{(13-15)}$. Therefore, DSS-induced colitis is a well-established model for testing the effects of the suggested agents.

Cytokines, the inflammatory mediators, have a key role in innate and adaptive immune reactions. They regulate immune cell functions that trigger signalling pathways and mediate proliferation and differentiation of immune cells in inflammatory diseases including colitis ${ }^{(15)}$. Cytokines can be classified along the helper T (Th)1, Th2, Th17 and regulatory $\mathrm{T}\left(\mathrm{T}_{\text {reg }}\right)$ cell spectra, and cytokines in each class are responsible for immune reactions specific to that subset ${ }^{(15)}$. Thus, it is important to identify the related cytokine changes according to the Th subsets. Acute DSS-induced colitis is considered analogous to Crohn's disease, which is known to involve the Th1/Th17/ $\mathrm{T}_{\text {reg }}$-dependent pathological process ${ }^{(16,17)}$.

Considering both the traditional use and bioactivities of LJ, we postulated that LJ could show preventive effects against colitis. In the present study, we investigated the effects of LJ in a DSSinduced IBD mouse model by assessing body weight, colon length and histological damage. Mechanistic studies were also conducted in vivo and in vitro by (1) assessing the promotion of specific $\mathrm{T}$ cell lineages, (2) profiling cytokine expression via a biometric multiplex signalling method and (3) analysing the prevalence of $\mathrm{CD}^{+} \mathrm{CD} 25^{+} \mathrm{Foxp} 3^{+} \mathrm{T}_{\text {reg }}$ cells.

\section{Materials and methods}

\section{Animals}

Male Balb/c mice (7 weeks old, 20-24g) were supplied by Daehan Bio Link for in vivo experiments. Foxp $3^{\text {EGFP }}$ Balb/c (C. Cg-Foxp3tm2Tch/J) mice were purchased from the Jackson Laboratory for in vitro experiments. Mice were housed at $20-22^{\circ} \mathrm{C}$ and in $40-60 \%$ humidity under a $12 \mathrm{~h}$ light $-12 \mathrm{~h}$ dark cycle, and were provided food and water ad libitum. All procedures were conducted according to the animal welfare guidelines of the National Institute of Health, and the present study was approved by the Kyung Hee University Institutional Animal Care and Use Committee (KHUASP(SE)-09-036).

\section{Sample preparation and HPLC analysis}

Flowers of LJ (Caprifoliaceae, $390 \mathrm{~g}$ ) were purchased from the Kyung Hee Hanyak Company, and extracts were prepared by boiling the flowers in water at $100^{\circ} \mathrm{C}$ for $2 \mathrm{~h}$. LJ water extract (LJE, yield 6.83\%) was obtained by evaporating and freeze-drying. LJ (PSO03) and LJE (PSE003) were deposited in the Department of Herbal Pharmacology at the College of Oriental Medicine, Kyung Hee University. For the HPLC analysis of LJE, $2 \mathrm{mg}$ of LJE were dissolved in $50 \%$ methanol ( $1 \mathrm{ml}$ ) and then filtered through a $0.22 \mu \mathrm{m}$ membrane filter (Millipore). Then, $10 \mu \mathrm{l}$ of the sample or standard (CGA (3-O-caffeoylquinic acid, $\mathrm{C}_{16} \mathrm{H}_{18} \mathrm{O}_{9}$ ), purity >95\%; SigmaAldrich) were injected into the HPLC system (Agilent Technologies). The sample was analysed on a Capcell Pak UG120 $\mathrm{C}_{18}$ analytical column $(150 \times 4.6 \mathrm{~mm}, 5 \mu \mathrm{m}$; Shiseido). The oven temperature was maintained at $35^{\circ} \mathrm{C}$. The mobile phase comprised $0 \cdot 1 \%$ formic acid (A) and acetonitrilemethanol-formic acid (0.1\%) (45:45:10) (B); the flow rate was $1 \mathrm{ml} / \mathrm{min}$. The gradient elution programme was used as follows: initially, $\mathrm{A}: \mathrm{B}=84 \%: 16 \%$, which was linearly changed to $\mathrm{A}: \mathrm{B}=60 \%: 40 \%$ at $25 \mathrm{~min}$, and finally maintained up to $27 \mathrm{~min}$. At $27.5 \mathrm{~min}$, the composition of the mobile phase returned to the initial conditions, which were maintained for $7.5 \mathrm{~min}$ for column re-equilibration. Chromatograms were acquired at $245 \mathrm{~nm}$ by UV detection. In the HPLC chromatogram, CGA (retention time $=11.3 \mathrm{~min}$ ) was found to be a major component of the extract sample. The content of CGA was 10.51 (SD 0.02)\% (Fig. 1).

\section{Induction of colitis and experimental design}

Colitis was induced by DSS as described previously ${ }^{(7)}$. Mice were provided with drinking-water containing 5\% DSS (MP Biomedicals) ad libitum for $7 \mathrm{~d}$. In the present study, we selected the dosages of LJE in consideration of standard human dosages. LJE at a dosage of $20 \mathrm{mg} / \mathrm{kg}$ in mice corresponds to $1.0 \mathrm{~g} \mathrm{LJE} / 50 \mathrm{~kg}$-weighed human subject, where LJE is extracted from approximately $14.7 \mathrm{~g}$ of the LJ raw material; this concentration is similar to the dosage of LJE in honeysuckle drinking tea $(10-15 \mathrm{~g} / \mathrm{d})^{(18)}$. Finally, we decided the dosages of LJE, i.e. 20, 100 and $500 \mathrm{mg} / \mathrm{kg}$, separated by five time intervals. In the case of CGA, as a main component of LJE, we calculated the dosage using HPLC analysis. We chose a dosage of $52.5 \mathrm{mg} / \mathrm{kg}$ of CGA, which corresponded to $500 \mathrm{mg} / \mathrm{kg}$ of LJE. To investigate the effects of the co-treatment of LJE, mice were randomly allocated into five groups ( $n$ 8): normal, DSS and three DSS + LJE (20, 100 and $500 \mathrm{mg} / \mathrm{kg}$ ) groups. To compare the effects of CGA $(52.5 \mathrm{mg} / \mathrm{kg})$ and LJE $(500 \mathrm{mg} / \mathrm{kg})$, mice were randomly allocated into four groups ( $n$ 8): normal, DSS, DSS + CGA $52.5 \mathrm{mg} / \mathrm{kg}$ and DSS + LJE $500 \mathrm{mg} / \mathrm{kg}$ groups. Mice were administered distilled water $(10 \mathrm{ml} / \mathrm{kg})$ in the DSS group or each dose of LJE in the DSS + LJE group by a feeding needle twice per day from day 1 to day 7 . To compare the effects of delayed treatment with the co-treatment of LJE, mice were randomly allocated into three groups $(n 8)$ : normal, DSS and DSS + LJE $500 \mathrm{mg} / \mathrm{kg}$ groups. Mice were administered distilled water $(10 \mathrm{ml} / \mathrm{kg})$ in the DSS group or LJE $500 \mathrm{mg} / \mathrm{kg}$ in the DSS + LJE $500 \mathrm{mg} / \mathrm{kg}$ group, twice per day from day 4 to day 7 . 


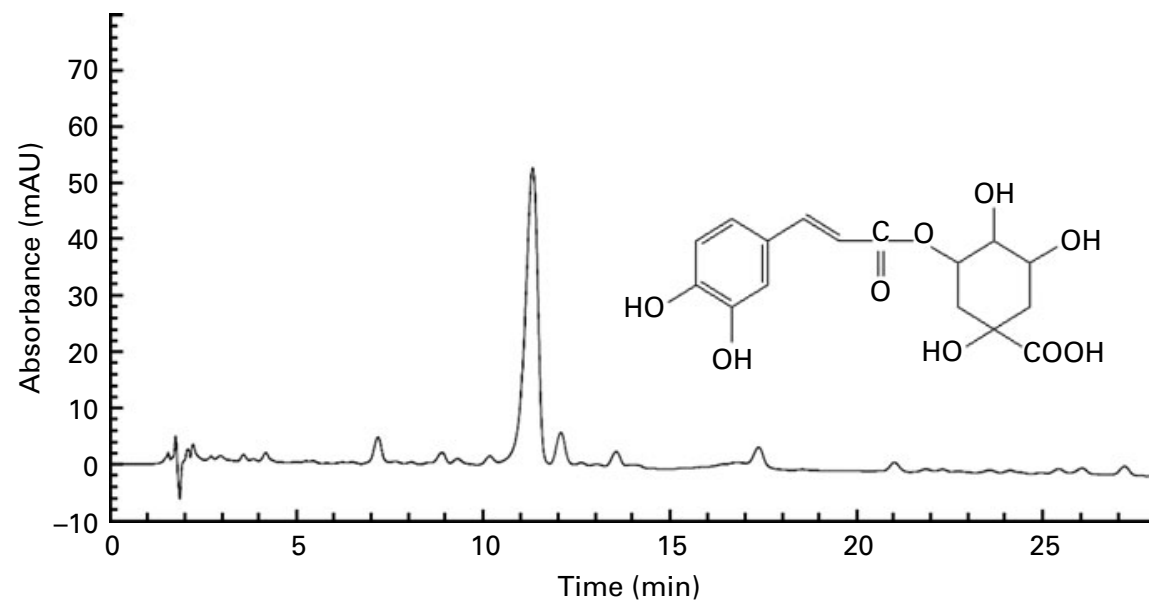

Fig. 1. A representative HPLC chromatogram of the water extract of Lonicera japonica. Chlorogenic acid (retention time $=11.3 \mathrm{~min}$ ). $\mathrm{AU}$, arbitrary units.

\section{Assessment of mouse weight and colon length}

Mouse weight was measured daily from day 0 to day 7 . The colon was isolated immediately after the last weight check. Colon length was measured from the caecum to the anus using a vernier caliper (Mitutoyo).

\section{Histological scoring}

Colons were fixed in $10 \%$ buffered formalin and embedded in paraffin. Histological sections cut from the paraffin blocks were stained with haematoxylin and eosin. In a blind fashion, the scoring of histological damage was divided into two categories: inflammatory cell infiltration and ulceration. Inflammatory cell infiltration was graded on a scale of $0-3(0=$ none; $1=$ mild $; 2=$ moderate; 3 = severe), obtained from each layer of the colon, including surface epithelium, cryptal glands, stroma, submucosa and transmural layer. The severity of ulceration was graded histologically on a scale of $0-4(0=$ none; $1=$ mild and focal surface; $2=$ mucosal layer; $3=$ submucosal layer; $4=$ transmural layer ${ }^{(7,19)}$. We modified and established a 0-19point system by using the sum of all scores.

\section{Collection of colonic mucosa and biometric multiplex cytokine profiling}

Isolated colons were snap-frozen and stored at $-70^{\circ} \mathrm{C}$. The mucosa was scraped from the muscle layer of the colon. Next, it was weighed, and $10 \mathrm{mg}$ mucosa were dissolved in triple-detergent lysis buffer $(50 \mathrm{~mm}$-Tris-HCl, $\mathrm{pH} 8.0$, $150 \mathrm{~mm}-\mathrm{NaCl}, 0 \cdot 1 \%$ SDS, $1 \% \mathrm{NP}-40,0.02 \%$ sodium azide, $0 \cdot 5 \%$ sodium deoxycholate and $1 \mathrm{~mm}$-phenylmethylsulfonyl fluoride) and homogenised. The levels of nine cytokines covering a broad spectrum of immune and inflammatory mechanisms were measured in parallel following the induction of colitis ${ }^{(16)}$. The cytokine levels (interferon (IFN)- $\gamma$, TNF- $\alpha$, IL-1 $\beta$, IL- 6 and IL-12 as Th1 panels; IL-10 and transforming growth factor (TGF)- $\beta 1$ as $\mathrm{T}_{\text {reg }}$ panels; IL-17 and IL-23 as Th17 panels) in the mucosa samples were analysed according to the manufacturer's manual of biometric multiplex cytokine assay (Millipore).

\section{Regulatory T cell measurement in dextran sulphate sodium-induced colitis}

To investigate the effect of LJE on $\mathrm{T}_{\text {reg }}$ cell promotion, mice were divided into four groups (non-treated normal group, LJE $500 \mathrm{mg} / \mathrm{kg}$ group, DSS group and DSS + LJE $500 \mathrm{mg} / \mathrm{kg}$ group; $n 8$ for each group). Spleens were isolated and ground over a wire mesh screen at day 7 of the DSS treatment. Erythrocytes were lysed in $0.85 \% \mathrm{NH}_{4}$ in Tris- $\mathrm{HCl}$ buffer. Next, splenocyte suspension was centrifuged at $300 \boldsymbol{g}$ for 5 min and resuspended in Roswell Park Memorial Institute 1640 medium (WelGENE, Inc.) supplemented with 10\% fetal bovine serum, penicillin $(30 \mu \mathrm{g} / \mathrm{ml})$ and streptomycin $(50 \mu \mathrm{g} / \mathrm{ml})$ (Hyclone). For evaluating $\mathrm{T}_{\text {reg }}$ populations, splenocytes were analysed by flow cytometry. Antibodies that were used for flow cytometry included fluorescein isothiocyanateconjugated anti-mouse CD4, R-phycoerythrin (PE)-conjugated anti-mouse CD25 and PE-Cy5 anti-mouse Foxp3 (eBioscience). Multiple-colour immunofluorescence staining was performed using a mouse $\mathrm{T}_{\text {reg }}$ staining kit (eBioscience) following the manufacturer's instructions. All samples were analysed on a FACSCalibur and CellQuest (BD Bioscience) and data were calculated as the percentage of $\mathrm{CD} 25^{+} \mathrm{Foxp} 3^{+}$cells among the total $\mathrm{CD}^{+}$cells.

\section{Fluorescence-activated cell sorting analysis for Foxp3} in vitro

The method of splenocyte isolation from Foxp $3^{\text {EGFP }}$ Balb/c mice has been described above. To examine the effect of LJE on $\mathrm{T}_{\text {reg }}$ cells in vitro, splenocytes were treated with LJE ( 1 and $10 \mu \mathrm{g} / \mathrm{ml}$ ), bee venom (a positive control, $1 \mu \mathrm{g} / \mathrm{ml}$; Sigma-Aldrich) or PBS in the presence of anti-CD3 $\varepsilon$ $(10 \mu \mathrm{g} / \mathrm{ml})$ and anti-CD28 anti-mouse monoclonal antibodies ( $2 \mu \mathrm{g} / \mathrm{ml}$ ) (BD Biosciences). After $72 \mathrm{~h}$, cells were stained for flow cytometric analyses. Antibodies that were used for flow cytometry included antigen-presenting cell-conjugated antimouse CD4 (eBioscience). The methods used for performing multiple-colour immunofluorescence staining and analysis were the same as those used in the in vivo study. Data were 
obtained and calculated by setting the DSS group as 100\% and expressing the remaining samples as a percentage of the DSS group.

\section{Statistical analysis}

All results are expressed as mean values with their standard errors for each group. Data were analysed statistically using one-way ANOVA followed by Dunnett's test for comparative analysis with the DSS group and by Tukey's test for comparative analysis between the groups. $P<0.05$ was regarded as statistically significant.

\section{Results}

\section{Effects of the water extract of Lonicera japonica on weight loss in dextran sulphate sodium-induced colitis}

Body weights of mice in the normal group continuously increased until day 7; however, the body weight of those in the DSS group continuously decreased. The DSS + LJE administration increased mouse weight in a dose-dependent manner compared with the DSS group. In particular, the DSS + LJE $500 \mathrm{mg} / \mathrm{kg}$ group presented a significant protective effect against weight loss induced by DSS on days 5, 6 and 7 $(P<0 \cdot 05$; Fig. 2).

\section{Effects of the water extract of Lonicera japonica on colon} shortening in dextran sulphate sodium-induced colitis

The colons from the normal group were thin and healthy with intact caeca. However, the colons from the DSS group were short, oedematous and hyperaemic, with damaged caeca. The DSS + LJE groups showed lesser short and oedematous colons with lesser-damaged caeca than the DSS group (Fig. 3(a)). The administration of $5 \%$ DSS for $7 \mathrm{~d}$ induced colon shortening in the DSS group compared with the

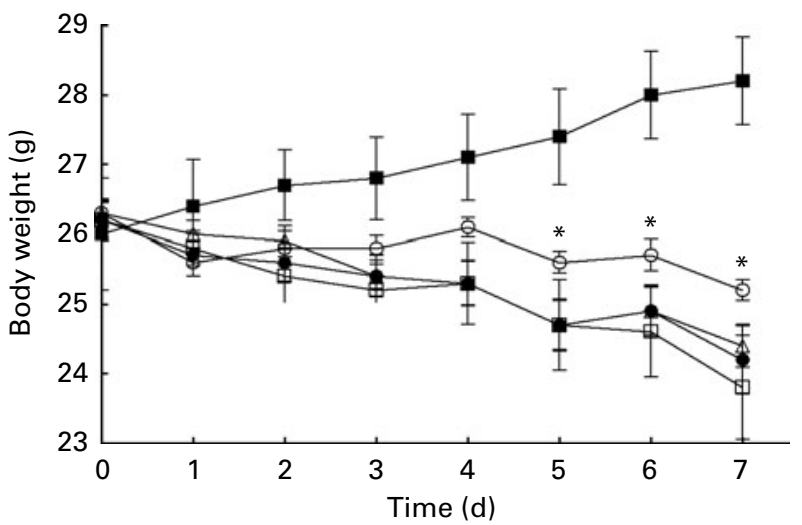

Fig. 2. Effects of the water extract of Lonicera japonica (LJE) on mouse weight in dextran sulphate sodium (DSS)-induced colitis. The normal group $(-)$ ) was not treated with DSS or LJE. Values are means ( $n 8$ for each group), with their standard errors represented by vertical bars. *Mean values were significantly different compared with the DSS group at each day $(P<0.05)$. DSS, group treated with $5 \%$ of DSS (-); DSS + LJE, DSS group treated with $20 \mathrm{mg} / \mathrm{kg}(\square), 100 \mathrm{mg} / \mathrm{kg}(\triangle)$ and $500 \mathrm{mg} / \mathrm{kg}(\bullet)$ of LJE. normal group. The LJE treatment significantly inhibited colon shortening in a dose-dependent manner $(P<0.05$ in the DSS $+\mathrm{LJE} 20 \mathrm{mg} / \mathrm{kg}$ group and $P<0.001$ in the DSS + LJE $100 \mathrm{mg} / \mathrm{kg}$ and $500 \mathrm{mg} / \mathrm{kg}$ groups; Fig. 3(b)).

\section{Effects of the water extract of Lonicera japonica on the} histological damage of colonic mucosa in dextran sulphate sodium-induced colitis

Surface epithelium, cryptal glands, stroma and submucosa from the normal group were intact, while the DSS group showed severe damage to the overall surface epithelium, disruption of the cryptal glands and infiltration of the inflammatory cells (Fig. 4(a) and (b)). The DSS + LJE groups showed protective effects against histological damage of the colonic mucosal layer induced by DSS. The DSS + LJE $20 \mathrm{mg} / \mathrm{kg}$ group showed a relatively intact surface epithelium compared with the DSS group; however, disruption of the cryptal glands and infiltration of the inflammatory cells were similar to those observed in the DSS group (Fig. 4(c)). The DSS + LJE $100 \mathrm{mg} / \mathrm{kg}$ group presented a more intact surface epithelium and cryptal glands than those in the DSS and DSS + LJE $20 \mathrm{mg} / \mathrm{kg}$ groups (Fig. 4(d)). In the DSS + LJE $500 \mathrm{mg} / \mathrm{kg}$ group, a small amount of inflammatory cell infiltration was found, while cryptal glands and surface epithelium were intact, which was similar to that observed in the normal group (Fig. 4(e)).

The histological score of the normal group was set to 0 points, and the DSS group scored over 10 points. However, the DSS + LJE groups showed dose-dependent protective effects against DSS-induced histological damage. The DSS + LJE $100 \mathrm{mg} / \mathrm{kg}$ and $500 \mathrm{mg} / \mathrm{kg}$ groups presented significant protection from histological damage $(P<0.05$ and $P<0 \cdot 01$, respectively; Fig. 4).

\section{Effects of the water extract of Lonicera japonica on cytokine levels in colonic mucosa}

To analyse the influence of LJE on cytokine production in colonic mucosa during colitis, we investigated representative Th1 (IFN- $\gamma$ ) and Th17 (IL-17 and IL-23) cytokine levels through multiplex profiling after $7 \mathrm{~d}$ of DSS administration. LJE inhibited IFN- $\boldsymbol{\gamma}$ in a dose-dependent manner $(P<0.05$ in the DSS + LJE $500 \mathrm{mg} / \mathrm{kg}$ group compared with the DSS group; Fig. 5). The level of IL-17 in the DSS group showed a significant increase compared with the normal group, and the LJE treatment reduced IL-17 levels in a dose-dependent manner. In particular, the level of IL-17 in the DSS + LJE $500 \mathrm{mg} / \mathrm{kg}$ group was as low as that in the normal group $(P<0.05 ; \quad$ Fig. 5). LJE also inhibited other Th1/Th17 response-related cytokines including TNF- $\alpha$, IL-1 $1 \beta$, IL- 6 and IL-12 in a dose-dependent manner (Fig. 5). IL-23, which is related to Th17 differentiation, IL-10 and TGF- $\beta 1$ did not show any significant differences in all groups (Fig. 5). 
(a)

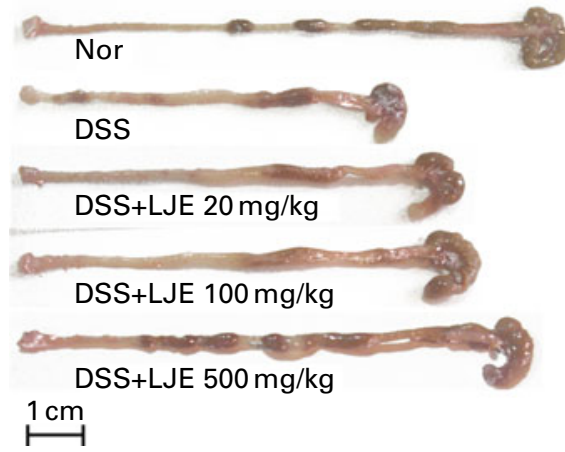

(b)

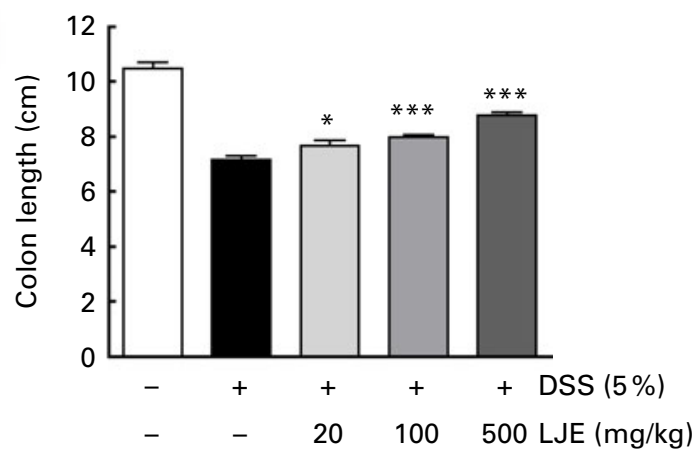

Fig. 3. Effects of the water extract of Lonicera japonica (LJE) on colon length in dextran sulphate sodium (DSS)-induced colitis. (a) Representative images of a typical colon morphology and the length of each group. (b) A graph showing the data of colon length. Values are means ( $n 8$ for each group), with their standard errors represented by vertical bars. Mean values were significantly different compared with the DSS group: $\left.{ }^{\star} P<0.05,{ }^{\star \star \star} P<0.001\right)$. Nor, normal group; DSS, a group treated with DSS; DSS + LJE, DSS group treated with each dosage of the water extract of Lonicera japonica (a colour version of this figure can be found online at www.journals.cambridge.org/bjn).

\section{Effects of the water extract of Lonicera japonica on regulatory $T$ cell populations in dextran sulphate sodium-induced colitis}

The administration of $500 \mathrm{mg} / \mathrm{kg}$ of LJE, as the effective dosage, for $7 \mathrm{~d}$ did not significantly increase $T_{\text {reg }}$ populations in the spleen compared with the non-treated normal group. While DSS administration increased $\mathrm{T}_{\text {reg }}$ populations $(P<0.05$; Fig. 6), the DSS $+\mathrm{LJE} 500 \mathrm{mg} / \mathrm{kg}$ group did not show any effect on $\mathrm{T}_{\text {reg }}$ populations compared with the DSS group (Fig. 6). To confirm the effects of LJE on $\mathrm{T}_{\text {reg }}$ populations, we performed fluorescence-activated cell sorting analysis for Foxp3 in vitro. $\mathrm{T}_{\text {reg }}$ populations in cells treated with bee venom, as a positive control, increased two times more than the DSS group. However, LJE $(1$ and $10 \mu \mathrm{g} / \mathrm{ml})$ did not increase $\mathrm{T}_{\text {reg }}$ populations in splenocyte cultures (Fig. 7).

\section{Effects of the delayed treatment of the water extract of Lonicera japonica on colon damage}

The delayed treatment of $500 \mathrm{mg} / \mathrm{kg}$ of LJE from day 4 to day 7 showed only $18.7 \%$ of the protective effect on colon shortening compared with the DSS group ( $P<0 \cdot 05$; Fig. 8$)$, which was far less effective than the co-treatment of $500 \mathrm{mg} / \mathrm{kg}$ of LJE ( $48.5 \%$ of protection; Fig. 2).

\section{Effects of the water extract of Lonicera japonica and chlorogenic acid on colon damage}

The treatment of $500 \mathrm{mg} \mathrm{LJE} / \mathrm{kg}$ and $52.5 \mathrm{mg} \mathrm{CGA} / \mathrm{kg}$ from day 1 to day 7 showed 40.8 and $24.9 \%$ of the protective effects against colon shortening compared with the DSS group, respectively $(P<0.001$ for the $L J E 500 \mathrm{mg} / \mathrm{kg}$ treatment,
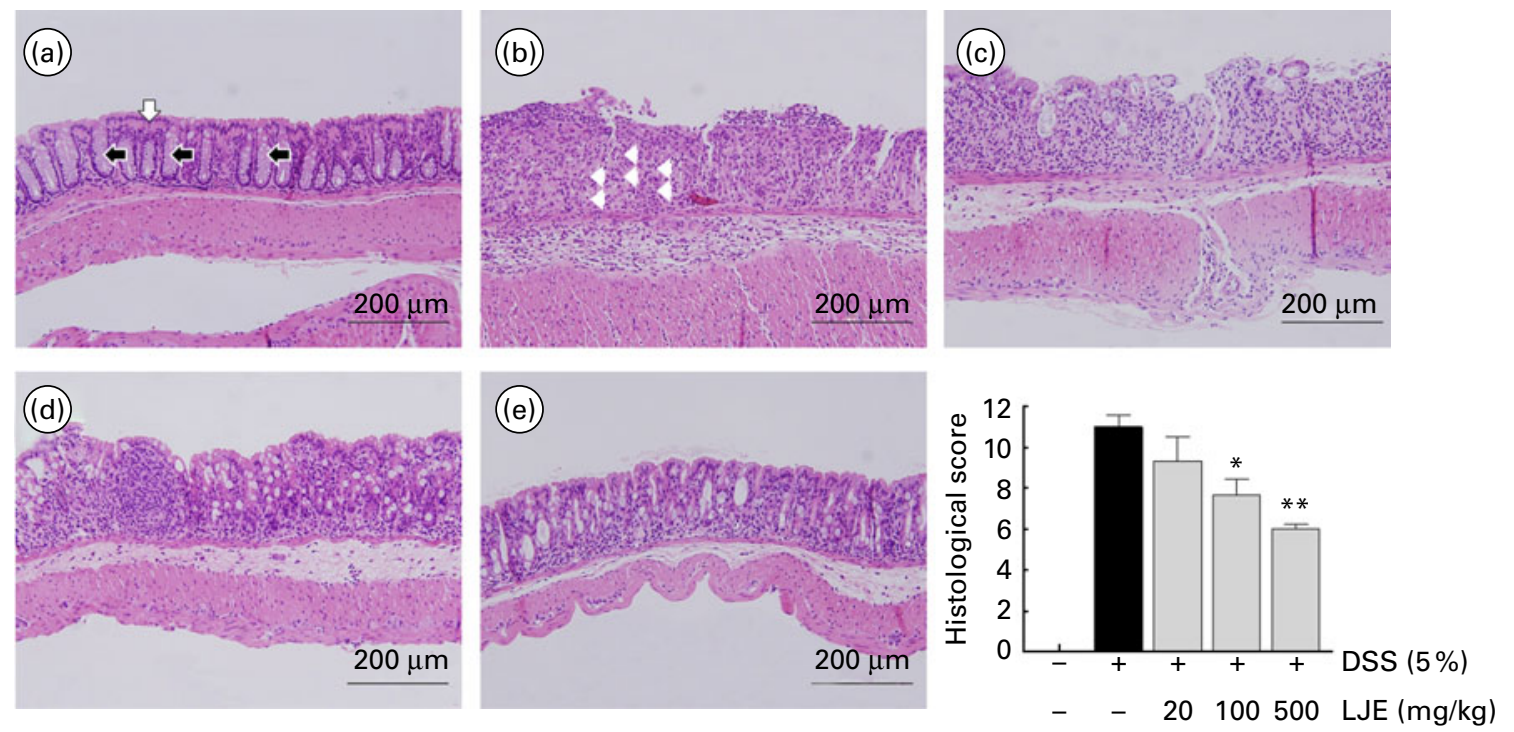

Fig. 4. Effects of the water extract of Lonicera japonica (LJE) on histological findings and score in dextran sulphate sodium (DSS)-induced colitis. The images $(\mathrm{a}-\mathrm{e})$ are the representative histological findings of all groups:(a) normal, (b) DSS, (c) DSS + LJE $20 \mathrm{mg} / \mathrm{kg}$, (d) DSS + LJE 100 mg/kg and (e) DSS + LJE $500 \mathrm{mg} / \mathrm{kg}$ groups. $\longleftarrow$, cryptal grand; $\{$, surface of the epithelium; $\triangleleft$, neutrophils. The graph shows the histological scores. The score of the normal group is zero. Values are means ( $n 8$ for each group), with their standard errors represented by vertical bars. Mean values were significantly different compared with the DSS group: ${ }^{*} P<0.05,{ }^{* *} P<0.01$ (a colour version of this figure can be found online at www.journals.cambridge.org/bjn). 

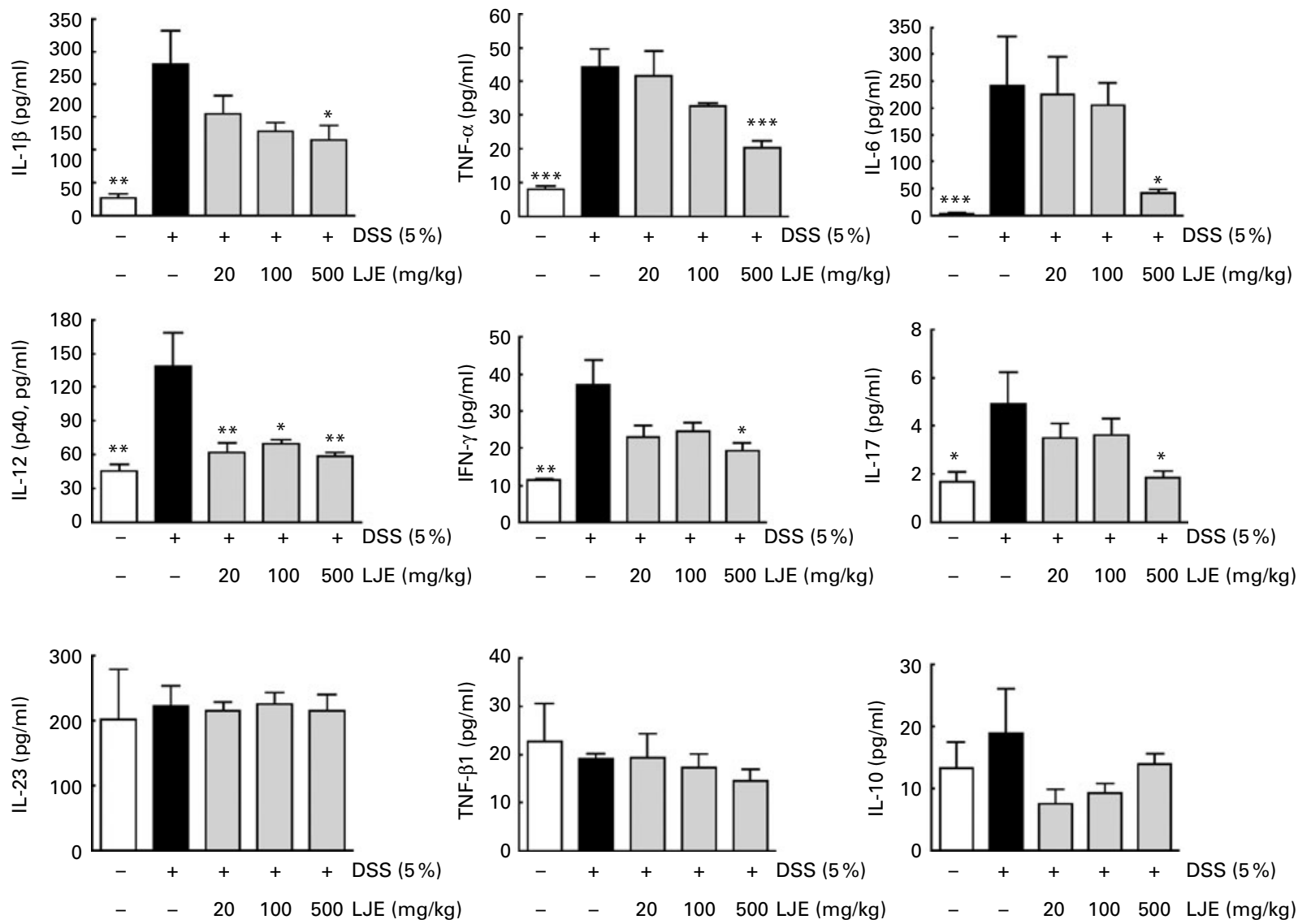

Fig. 5. Effects of the water extract of Lonicera japonica (LJE) on cytokine production induced by dextran sulphate sodium (DSS) in mouse colonic mucosa. Values are means ( $n 8$ for each group), with their standard errors represented by vertical bars. Mean values were significantly different compared with the DSS group: ${ }^{*} P<0.05,{ }^{* *} P<0.01,{ }^{* * *} P<0.001$. IFN- $\gamma$, interferon- $\gamma ;$ TGF- $\beta 1$, transforming growth factor- $\beta 1$.

$P<0.01$ for the CGA $52.5 \mathrm{mg} / \mathrm{kg}$ treatment; Fig. 8(b)). The LJE $500 \mathrm{mg} / \mathrm{kg}$ treatment showed more protective effect than the CGA $52.5 \mathrm{mg} / \mathrm{kg}$ treatment $(P<0.05$; Fig. $8(\mathrm{~b}))$.

\section{Discussion}

In the present study, oral administration of LJE at a dose of 20, 100 and $500 \mathrm{mg} / \mathrm{kg}$ showed dose-dependent inhibitory effects against colon shortening, weight loss and histological damage associated with DSS-induced colitis. LJE down-regulated Th1/Th17 pathway-related cytokines. However, IL-10, IL-23 and TGF- $\beta 1$ were not affected. LJE also did not show any significant effects on $\mathrm{T}_{\text {reg }}$ populations. The effect of the delayed treatment of LJE was less effective than that of the co-treatment of LJE.

LJE administration inhibited the shortening of colon length in a dose-dependent manner, and the maximal protective effect of LJE was achieved at a dose of $500 \mathrm{mg} / \mathrm{kg}$. In general, DSS administration in mice is known to induce colitis similar to that observed in human IBD. Oral administration of DSS, a heparin-like polysaccharide, induces inflammatory structural changes, which have been reported to be initiated by direct damage to gut epithelial cells of the basal crypts and subsequent damage of the mucosal barrier integrity ${ }^{(19,20)}$. Lumen bacteria produce inflammatory cytokines including
IL-6 and TNF- $\alpha$ at the damaged site, and cause colonic mucosal inflammation, erosion and ulceration. As a result of these inflammatory reactions, the colon is known to be shortened $^{(19,20)}$. The effects of LJE on colon shortening might be supported by the results from the present study, which revealed that LJE treatment protected against DSS-induced cryptal gland loss and epithelial damage and inhibited inflammatory cytokines. Therefore, it could be suggested that LJE showed anti-inflammatory and preventive potential by inhibiting initial structural damage to the intestine in DSS-induced colitis. Inflammatory structural changes in a DSS-induced colitis model lead to various clinical symptoms such as diarrhoea, bloody stool, weight loss and behavioural changes ${ }^{(13-15,21)}$. Among these symptoms, weight loss is considered one of the major systemic symptoms due to colonic structural damage ${ }^{(13,21)}$. In previous studies, mice treated with DSS for $7 \mathrm{~d}$ tended to rapidly lose weight from day $4^{(22,23)}$. The present results showed a similar pattern of weight loss in the DSS group. Hence, significant inhibition of weight loss by $500 \mathrm{mg} / \mathrm{kg}$ of LJE administration from day 4 might arise from a protective effect of LJE against structural damage and functional insufficiency of the inflamed colon induced by DSS.

To evaluate the effects of samples on IBD in in vivo models, two kinds of protocols can be used: one protocol is pre- or co-treatment of the sample with DSS to investigate 

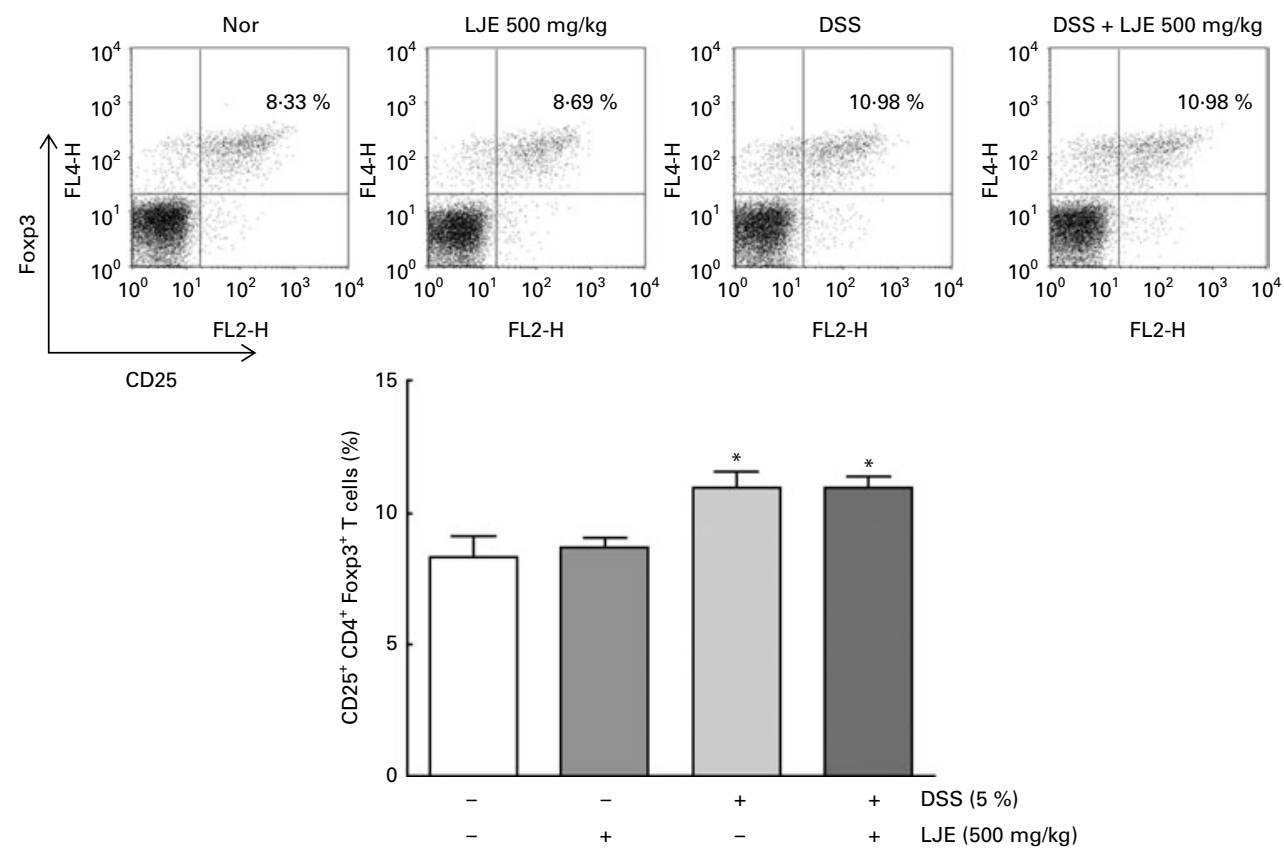

Fig. 6. Effects of the water extract of Lonicera japonica (LJE) on $\mathrm{CD} 25^{+} \mathrm{CD} 4^{+} \mathrm{Foxp} 3^{+} \mathrm{T}$ cell populations in dextran sulphate sodium (DSS)-induced mouse colitis The upper images indicate representative flow cytometry analysis of splenocytes in each group. The graph shows the data of flow cytometry analysis in each group $(n 8)$. Values are means, with their standard errors represented by vertical bars. * Mean values were significantly different compared with the DSS group $(P<0.05)$. Nor, normal group; LJE $500 \mathrm{mg} / \mathrm{kg}$, group treated with $500 \mathrm{mg} / \mathrm{kg}$ of LJE without DSS treatment; DSS, group treated with DSS from day 1 to day 7 ; DSS + LJE $500 \mathrm{mg} / \mathrm{kg}$, DSS group treated with $500 \mathrm{mg} / \mathrm{kg}$ of LJE; FL2-H, fluorescence intensity 2-height; FL4-H, fluorescence intensity 4-height.

the preventive effects, while the other is delayed treatment to investigate the therapeutic effects ${ }^{(24,25)}$. In the present study, day 4 might be the time point at which structural changes caused by DSS administration exceeded a critical point, disturbing normal intestinal function and leading to systemic symptoms. In the delayed-treatment design (LJE administration from day 4 to day 7 of the DSS treatment), the LJE treatment was less effective than the co-treatment
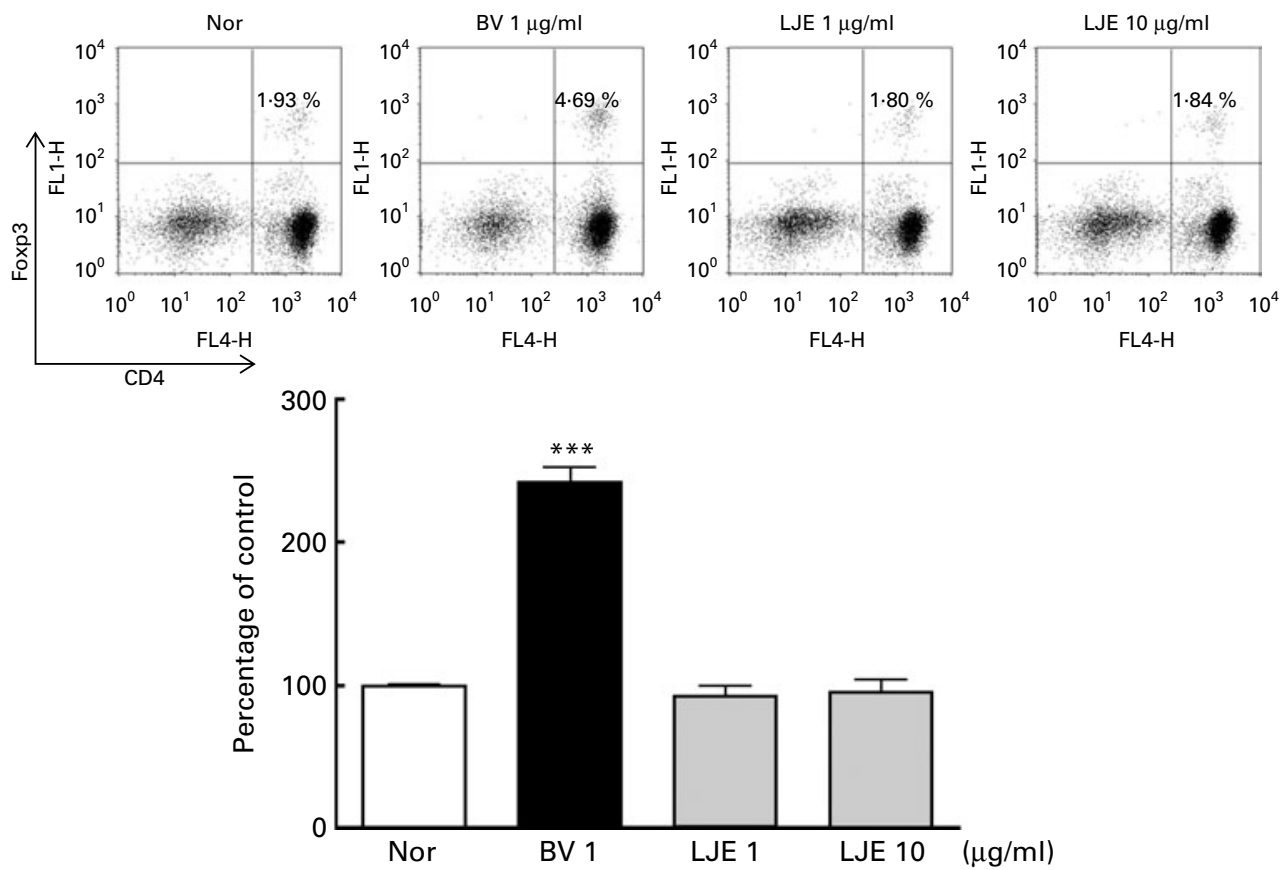

Fig. 7. Effects of the water extract of Lonicera japonica (LJE) on CD4 ${ }^{+}$Foxp $3^{+} \mathrm{T}$ cell populations in vitro. The upper images indicate representative flow cytometry analysis of splenocytes in each group. The graph shows the data of flow cytometry analysis in each group (in triplicate). BV, bee venom (1 $\mu \mathrm{g} / \mathrm{ml})$. Data of the dextran sulphate sodium (DSS) group was set at $100 \%$, and data of the other groups were calculated and expressed as a percentage of the DSS group. Values are means, with their standard errors represented by vertical bars. ${ }^{\star \star \star}$ Mean value was significantly different compared with the DSS group $(P<0.001)$. Nor, normal group; BV $1 \mu \mathrm{g} / \mathrm{ml}$, group treated with $1 \mu \mathrm{g} / \mathrm{ml}$ of BV; LJE $1 \mu \mathrm{g} / \mathrm{ml}$, group treated with $1 \mu \mathrm{g} / \mathrm{ml}$ of LJE; LJE $10 \mu \mathrm{g} / \mathrm{ml}$, group treated with $10 \mu \mathrm{g} / \mathrm{ml}$ of LJE; FL1-H, fluorescence intensity 1-height; FL4-H, fluorescence intensity 4-height. 

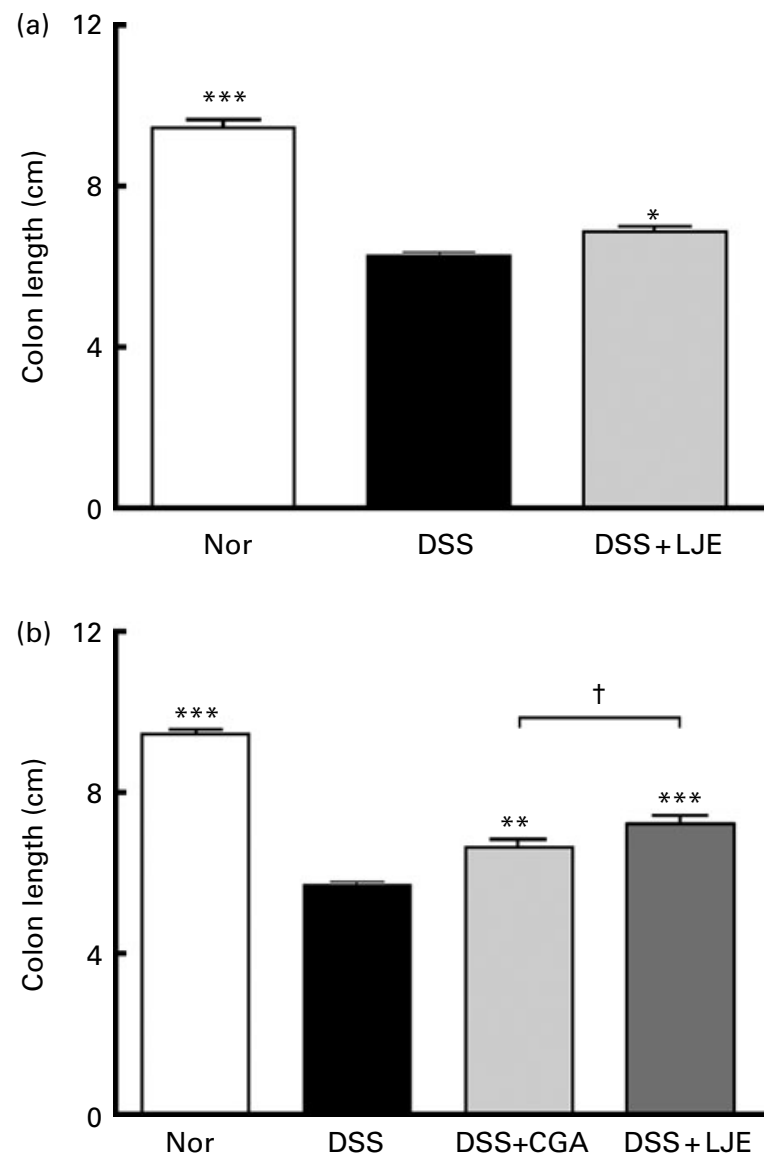

Fig. 8. Effect of the delayed treatment of (a) the water extract of Lonicera japonica (LJE) $(500 \mathrm{mg} / \mathrm{kg})$ and (b) the comparison between LJE $(500 \mathrm{mg} / \mathrm{kg})$ and chlorogenic acid (CGA) $(52.5 \mathrm{mg} / \mathrm{kg})$ on colon length in dextran sulphate sodium (DSS)-induced mouse colitis. Values are means ( $n 8$ for each group), with their standard errors represented by vertical bars. Mean values were significantly different compared with the DSS group: ${ }^{*} P<0.05$, ${ }^{* *} P<0.01$ ${ }^{* * *} P<0.001$. $†$ Mean values were significantly different from each other. Nor, normal group; DSS, group treated with DSS from day 1 to day 7; DSS + CGA, DSS group treated with $52.5 \mathrm{mg} / \mathrm{kg}$ of CGA from day 1 to day 7 DSS + LJE, DSS group treated with $500 \mathrm{mg} / \mathrm{kg}$ of LJE from day 4 to day 7 (a) and from day 1 to day 7 (b)

beginning at day 1 . In addition, allowing the sample to mix freely with the diet is a common method for investigating the effects of the food ingredients ${ }^{(26,27)}$. In the present study, LJE was administered orally by a feeding tube to investigate the effective dosage more precisely.

From the present results, it is noteworthy that $20 \mathrm{mg} / \mathrm{kg}$ of LJE, known as the general human dosage, also showed the protective effects on colitis, and the maximal effect of LJE on colitis was $500 \mathrm{mg} / \mathrm{kg}$ (although we did not present the data, the DSS + LJE $250 \mathrm{mg} / \mathrm{kg}$ group (LJE concentration 12.5 times the normal human dosage) showed 8.5 (SD 0.29) $\mathrm{cm}$ of colon length, which was similar to that observed in the DSS + LJE $500 \mathrm{mg} / \mathrm{kg}$ group). Since the metabolic capacity of mice has been reported to be about 10-fold that of humans, a safe and maximal effective dosage might be approximately $200 \mathrm{mg} / \mathrm{kg}$, and the present results are supported by these facts ${ }^{(28)}$. Therefore, the levels of effective dosages in the present study correspond to possible dosages to be administered in humans.

The main components of LJE in the present study are CGA ( $10.5 \%$ of LJE), an ester of caffeic acid and quinic acid, which is a well-known compound that has antioxidant ${ }^{(29)}$, anti-inflammatory $^{(8)}$, anti-carcinogenic ${ }^{(10)}$, analgesic ${ }^{(11)}$ and neuroprotective ${ }^{(12)}$ effects. Along with $\mathrm{LJ}$, it is also present in blueberries $^{(30)}$ and coffee $^{(31)}$. Recently, coffee has been reported to have anti-carcinogenic effects on various colon cancer models, and the major active components are CGA and its metabolites (comprising up to $12 \%$ of CGA in coffee), which have also been reported to have protective effects on colitis models ${ }^{(32-37)}$. In the present results, we also found that CGA showed protective effects on the DSS-induced colitis mice model, and that the LJE $500 \mathrm{mg} / \mathrm{kg}$ treatment showed more protective effect than the CGA $52.5 \mathrm{mg} / \mathrm{kg}$ treatment that corresponds to $500 \mathrm{mg} / \mathrm{kg}$ of LJE. Thus, the protective effects of LJE in the present study might arise from the additional effects of the components of LJE along with CGA.

In the present study, LJE treatment generated a dose-dependent decrease in histological score that reflects cryptal damages and the severity and extent of inflammation ${ }^{(19)}$. DSS administration induces direct gut epithelium damage and affects the integrity of the mucosal barrier ${ }^{(21)}$, which result in severe cryptal depletion, epithelial cell damage, ulceration of mucosa and submucosa, inflammatory cell infiltration, muscle thickening and tissue oedema ${ }^{(13)}$. Thus, the protective effects of LJE might result from the inhibitory effects against inflammation-induced structural changes.

Deleterious effects of pro-inflammatory cytokines in IBD have been well documented ${ }^{(15)}$. Thus, cytokine inhibition could be the target of the mechanistic studies of IBD; recent studies on IBD have focused on agents that can block cytokine production ${ }^{(15)}$. In particular, analysis of specific cytokine changes related to various Th-subset pathways could provide key mechanisms for the protective effects of putative therapeutic agents in colitis. Therefore, we tried to elucidate the effects of LJE on Th subset-specific cytokine production after the DSS treatment. A biometric multiplex cytokine signalling method was applied to analyse a broad spectrum of cytokines in colonic mucosa ${ }^{(16)}$. The DSS treatment in the present study up-regulated pro-inflammatory cytokines and Th1- and Th17related cytokines in inflamed mucosa, in accordance with recent studies ${ }^{(16)}$. LJE inhibited representative Th1 (IFN- $\gamma$ ) and Th17 (IL-17) cytokines, and Th1/Th17 response-related pro-inflammatory cytokines including TNF- $\alpha$, IL-1 $\beta$, IL-6 and IL-12, but not IL-23, in a dose-dependent manner, in colonic mucosa. Th17 has been reported to be one of the most deleterious immune cells that orchestrate tissue inflammation by inducing pro-inflammatory cytokines, chemokines and matrix metalloproteases, and by subsequently recruiting neutrophils or other Th1 cells to the target tissue ${ }^{(38,39)}$. Th1 cells are similar to most key immune cells in that they induce infiltration and activation of effector T cells or mononuclear cells, which results in direct tissue damage through the massive production of inflammatory cytokines, including IFN- $\gamma^{(15,38,39)}$. Thus, it could be suggested that the severity of histological damage in colitis might be closely related to the activation 
of the Th1/Th17 pathway. The present results showing the inhibitory effects of LJE on Th1/Th17 cytokines (IFN- $\gamma$ and IL-17), tissue damage of colonic mucosa and neutrophil infiltration could suggest that LJE attenuates colitic damage by modulating Th1/Th17 lineage development. Previous studies of caffeic acid in DSS-induced colitis have shown this compound to inhibit inflammatory damage to the colon and decrease $I L-17$ gene expression ${ }^{(36)}$. These findings are in agreement with the present results and could indicate that the protective effects of LJE may originate from CGA metabolites.

In the present study, LJE did not inhibit IL-23 production in spite of Th17 inhibition. In general, IL-23 plays an important role in the differentiation of naive $\mathrm{CD}^{+}{ }^{+} \mathrm{T}$ cells into $\mathrm{Th} 17^{(15)}$. However, the function of IL-23 on Th17 differentiation is exhibited only in the presence of IL- 6 and TGF- $\beta 1^{(10,15,40)}$. Moreover, it has also been reported to involve in only sustained IL-17 production and promote effector function in the chronic stage of colitis and not to be a necessary factor in the early commitment to Th17 lineage development ${ }^{(15,23)}$. Thus, the present results suggest that the Th17-inhibitory effect of LJE might be caused by factors other than IL-23.

In the present study, LJE did not show any inhibitory effects on IL-10, TGF- $\beta 1$ or $\mathrm{T}_{\text {reg }}$ promotion. IL-10 attenuates experimental colitis (and other $\mathrm{T}$ cell-mediated inflammatory responses) and induces $\mathrm{T}_{\text {reg }}$ populations ${ }^{(41)}$. TGF- $\beta 1$ is known to be secreted by $\mathrm{T}_{\text {reg }}$ cells and plays an important role in the activation and differentiation of $\mathrm{T}_{\text {reg }}$ cells, in conjunction with IL-6; $\mathrm{T}_{\text {reg }}$ cells then act as anti-inflammatory and immunomodulatory cells ${ }^{(41)}$. IL-10 and TGF- $\beta 1$ coordinately regulate homeostatic mucosal immune responses. IL10 has been shown to facilitate the expression of TGF- $\beta$ secreting $\mathrm{T}_{\text {reg }}$ cells and maintain Foxp3 expression and regulatory activity in an inflammatory milieu ${ }^{(41,42)}$. IL-10 and TGF- $\beta 1$ expression in the DSS group were not significantly higher than those in the normal group. Further, the levels of IL-10 and TGF- $\beta 1$ in the acute or subacute stages of colitis after DSS administration were not significantly elevated ${ }^{(16,42)}$. Therefore, the anti-inflammatory effects of LJE in the present study do not depend on increased IL-10 and TGF- $\beta 1$ in colonic mucosa, which indicates that the anti-inflammatory effects of LJE may not be related to $\mathrm{T}_{\text {reg }}$ cell differentiation or activation. The effects of LJE on these cytokines and $\mathrm{T}_{\text {reg }}$ cells are in parallel. $T_{\text {reg }}$ cell differentiation was more enhanced in the DSS group than in the normal group, and LJE administration did not show any inhibitory effects in the in vivo and in vitro studies. Therefore, the effects of LJE on DSS-induced colitis might not be due to $\mathrm{T}_{\text {reg }}$ cell-mediated immunomodulation.

In the present results, the inhibitory effects of LJE on the production of whole pro-inflammatory cytokines in colitis could be considered to be the immunosuppressive effects such as corticosteroids. Corticosteroids, including dexamethasone and methylprednisolone, have been reported to show immunosuppressive and protective effects in experimental colitis by enhancing the $\mathrm{T}_{\text {reg }}$ population ${ }^{(43,44)}$. Thus, the fact that LJE does not enhance $T_{\text {reg }}$ populations suggests that the effects of LJE are not steroid-like.
In conclusion, LJE showed protective effects against DSSinduced colitis via the Th1/Th17 pathway and not via $\mathrm{T}_{\text {reg }}$ cell-related mechanisms. This suggests the potential for using LJE as a preventive application against intestinal inflammation. Further investigation including studies of the chronic phase of inflammation is needed.

\section{Acknowledgements}

This study was supported by the Basic Science Research Program through the National Research Foundation of Korea funded by the Ministry of Education, Science and Technology (no. 2011-0013837 and 2005-0049404). J.-W. P., H. B., B. R. and Y. B. designed the study protocol. J.-W. P., H. B., B. R., K. L., Y. B., G. L, H. H. Y., B.-G. H. and J. B. conducted the in vivo and in vitro experiments. S.-J. L., B.-J. L. and J. K. performed the histological experiments and analysed the data. J.-W. P., H. B., H. L. and Y. B. wrote the draft of the manuscript. All authors read and approved the final manuscript. The authors declare that there is no conflict of interest.

\section{References}

1. Ahuja V \& Tandon RK (2010) Inflammatory bowel disease in the Asia-Pacific area: a comparison with developed countries and regional differences. J Dig Dis 11, 134-147.

2. Siegal CA (2011) Review Article: explaining risks of inflammatory bowel disease therapy to patients. Aliment Pharmacol Ther 33, 23-32.

3. Hou JK, Abraham B \& El-Serag H (2011) Dietary intake and risk of developing inflammatory bowel disease: a systematic review of the literature. Am J Gastroenterol 106, 563-573.

4. Lee JY, Kang HS, Park BE, et al. (2009) Inhibitory effects of Geijigajakyak-Tang on trinitrobenzene sulfonic acid-induced colitis. J Ethnopharmacol 126, 244-251.

5. Naito Y, Takagi T, Katada K, et al. (2006) Partially hydrolyzed guar gum down-regulates colonic inflammatory response in dextran sulfate sodium-induced colitis in mice. $J$ Nutr Biochem 17, 402-409.

6. Xu Y, Oliverson BG \& Simmons DL (2007) Trifunctional inhibition of COX-2 by extracts of Lonicera japonica: direct inhibition, transcriptional and post-transcriptional down regulation. J Ethnopharmacol 11, 667-670.

7. Ryu B, Ro W, Park JW, et al. (2011) Bojanggunbi-tang, a traditional Korean herbal prescription, ameliorates colonic inflammation induced by dextran sulfate sodium and 2,4,6trinitrobenzene sulfonic acid in mice. J Ethnopharmacol 135, 582-585.

8. Lee JP, Li YC, Chen HY, et al. (2010) Protective effects of luteolin against lipopolysaccharide-induced acute lung injury involves inhibition of MEK/ERK and PI3K/Akt pathways in neutrophils. Acta Pharmacol Sin 31, 831-838.

9. Suh SJ, Jin UH, Kim SH, et al. (2006) Ochnaflavone inhibits TNF-alpha-induced human VSMC proliferation via regulation of cell cycle, ERK1/2, and MMP-9. J Cell Biochem 99, 1298-1307.

10. Leung HW, Hour MJ, Chang WT, et al. (2008) P38-associated pathway involvement in apoptosis induced by photodynamic therapy with Lonicera japonica in human lung squamous carcinoma $\mathrm{CH} 27$ cells. Food Chem Toxicol 46 3389-3400. 
11. Yoo HJ, Kang HJ, Song YS, et al. (2008) Anti-angiogenic, antinociceptive and anti-inflammatory activities of Lonicera japonica extract. J Pharm Pharmacol 60, 779-786.

12. Kwon SH, Hong SI, Kim JA, et al. (2011) The neuroprotective effects of Lonicera japonica THUNB. against hydrogen peroxide-induced apoptosis via phosphorylation of MAPKs and PI3K/Akt in SH-SY5Y cells. Food Chem Toxicol 49, 1011-1019.

13. Kawada M, Arihiro A \& Mizoguchi E (2007) Insights from advances in research of chemically induced experimental models of human inflammatory bowel disease. World J Gastroenterol 13, 5581-5593.

14. Huang TC, Tsai SS, Liu LF, et al. (2010) Effect of Arctium lappa $\mathrm{L}$. in the dextran sulfate sodium colitis mouse model. World J Gastroenterol 16, 4193-4199.

15. Strober W \& Fuss IJ (2011) Proinflammatory cytokines in the pathogenesis of inflammatory bowel diseases. Gastroenterology 140, 1756-1767.

16. Alex P, Zachos NC, Nguyen T, et al. (2009) Distinct cytokine patterns identified from multiplex profiles of murine DSS and TNBS-induced colitis. Inflamm Bowel Dis 15, 341-352.

17. Lee IA, Bae EA, Hyun YJ, et al. (2010) Dextran sulfate sodium and 2,4,6-trinitrobenzene sulfonic acid induce lipid peroxidation by the proliferation of intestinal gram-negative bacteria in mice. J Inflamm 7, 7 .

18. Wu J (2005) An Illustrated Chinese Materia Medica pp. 398-399. New York, NY: Oxford University Press, Inc.

19. Kitajima S, Takuma S \& Morimoto M (2000) Histological analysis of murine colitis induced by dextran sulfate sodium of different molecular weights. Exp Anim 49, 9-15.

20. Cooper HS, Murthy SN, Shah RS, et al. (1993) Clinicopathologic study of dextran sulfate sodium experimental murine colitis. Lab Invest 69, 238-249.

21. Egger B, Bajaj-Elliott M, MacDonald TT, et al. (2000) Characterisation of acute murine dextran sodium sulphate colitis: cytokine profile and dose dependency. Digestion 62, $240-248$.

22. Ito R, Kita M, Shin-Ya M, et al. (2008) Involvement of IL-17A in the pathogenesis of DSS-induced colitis in mice. Biochem Biophys Res Commun 377, 12-16.

23. Im E, Choi YJ, Pothoulakis C, et al. (2009) Bacillus polyfermenticus ameliorates colonic inflammation by promoting cytoprotective effects in colitic mice. J Nutr 139, 1848-1854.

24. Karrasch T, Kim JS, Jang BI, et al. (2007) The flavonoid luteolin worsens chemical-induced colitis in NF-kappaB ${ }^{\text {EGFP }}$ transgenic mice through blockade of NF-kappaB-dependent protective molecules. PLoS One 2, e596.

25. Nam SY, Kim JS, Kim JM, et al. (2008) DA-6034, a derivative of flavonoid, prevents and ameliorates dextran sulfate sodium-induced colitis and inhibits colon carcinogenesis. Exp Biol Med 233, 180-191.

26. Vicario M, Amat C, Rivero M, et al. (2007) Dietary glutamine affects mucosal functions in rats with mild DSS-induced colitis. J Nutr 137, 1931-1937.

27. Spagnuolo PA \& Hoffman-Goetz L (2008) Dietary lactoferrin does not prevent dextran sulfate sodium induced murine intestinal lymphocyte death. Exp Biol Med 233, 1099-1108.

28. Chiou WL (1994) Determination of drug permeability in a flat or distended stirred intestine. Prediction of fraction dose absorbed in humans after oral administration. Int I Clin Pharmacol Ther 32, 474-482.

29. Choi CW, Jung HA, Kang SS, et al. (2007) Antioxidant constituents and a new triterpenoid glycoside from Flos Lonicerae. Arch Pharm Res 30, 1-7.

30. Gavrilova V, Kajdzanoska M, Gjamovski V, et al. (2011) Separation, characterization and quantification of phenolic compounds in blueberries and red and black currants by HPLC-DAD-ESI-MSn. J Agric Food Chem 59, 4009-4018.

31. Williamson G, Dionisi F \& Renouf M (2011) Flavanols from green tea and phenolic acids from coffee: critical quantitative evaluation of the pharmacokinetic data in humans after consumption of single doses of beverages. Mol Nutr Food Res 55, 864-873.

32. Kang NJ, Lee KW, Kim BH, et al. (2011) Coffee phenolic phytochemicals suppress colon cancer metastasis by targeting MEK and TOPK. Carcinogenesis 32, 921-928.

33. Saruwatari A, Isshiki M \& Tamura H (2008) Inhibitory effects of various beverages on the sulfoconjugation of 17beta-estradiol in human colon carcinoma Caco-2 cells. Biol Pharm Bull 31, 2131-2136.

34. Ferruzzi MG (2010) The influence of beverage composition on delivery of phenolic compounds from coffee and tea. Physiol Behav 100, 33-41.

35. Islam MS, Murata T, Fujisawa M, et al. (2008) Anti-inflammatory effects of phytosteryl ferulates in colitis induced by dextran sulphate sodium in mice. BrJ Pharmacol 154, 812-824.

36. Ye Z, Liu Z, Henderson A, et al. (2009) Increased CYP4B1 mRNA is associated with the inhibition of dextran sulfate sodium-induced colitis by caffeic acid in mice. Exp Biol Med 234, 605-616.

37. Oku H, Ogawa Y, Iwaoka E, et al. (2011) Allergy-preventive effects of chlorogenic acid and iridoid derivatives from flower buds of Lonicera japonica. Biol Pharm Bull 34, $1330-1333$.

38. Dardalhon V, Korn T, Kuchroo VK, et al. (2008) Role of Th1 and Th17 cells in organ-specific autoimmunity. J Autoimmun 31, 252-256.

39. Liu ZJ, Yadav PK, Su JL, et al. (2009) Potential role of Th17 cells in the pathogenesis of inflammatory bowel disease. World J Gastroenterol 15, 5784-5788.

40. Abraham C \& Cho J (2009) Interleukin-23/Th17 pathways and inflammatory bowel disease. Inflamm Bowel Dis 15, 1090-1100.

41. Boden EK \& Snapper SB (2008) Regulatory T cells in inflammatory bowel disease. Curr Opin Gastroenterol 24, 733-741.

42. Chen J, Xie L, Toyama S, et al. (2011) The effects of Foxp3expressing regulatory T cells expanded with CD28 superagonist antibody in DSS-induced mice colitis. Int Immunopharmacol 11, 610-617.

43. Prado C, Gómez J, López P, et al. (2011) Dexamethasone upregulates FOXP3 expression without increasing regulatory activity. Immunobiology 216, 386-392.

44. Zen M, Canova M, Campana C, et al. (2011) The kaleidoscope of glucorticoid effects on immune system. Autoimmun Rev 10, 305-310. 